\title{
Generalized cystic lymphangiomatosis
}

\author{
Darshana D. Rasalkar • Winnie C. W. Chu
}

Received: 29 December 2009/Revised: 29 March 2010 / Accepted: 3 April 2010 /Published online: 24 June 2010

(C) Springer-Verlag 2010

A 2-year-old girl presented with acute shortness of breath and multiple subcutaneous nodules. Chest radiograph showed widening of the mediastinum. Contrast-enhanced $\mathrm{CT}$ of the thorax revealed interstitial thickening, lung nodules, pleural effusions (Fig. 1a, black arrow) and cystic mediastinal collections (Fig. 1a, white arrow). Sections of the abdomen showed ascites, multiple lowdensity lesions in the spleen (Fig. 1b, black arrow) and retroperitoneum (Fig. 1b, white arrow). No appreciable enhancement was noted following administration of contrast medium. Multiple osteolytic lesions with sclerotic margins were also detected in the vertebral bodies (Fig. 1b, black arrowhead). Drainage of the pleural effusions revealed chylothorax.

Generalized lymphangiomatosis is a rare disease, characterized by a diffuse proliferation of lymphatic vessels that can involve any organ except the central nervous system [1]. Prognosis depends on the site and extent of the lymphangioma [1]. Imaging features are characteristic and crucial in the evaluation of the complete extent of disease and, thus, may contribute to clinical management of the disease by preventing initial misdiagnosis [2].

Electronic supplementary material The online version of this article (doi:10.1007/s00247-010-1694-7) contains supplementary material, which is available to authorized users.

\section{D. Rasalkar •W. C. W. Chu ( $\square)$}

Departments of Diagnostic Radiology and Organ Imaging,

The Chinese University of Hong Kong, Prince of Wales Hospital,

Ngan Shing St.,

Shatin, New Territories, Hong Kong

e-mail: winnie@med.cuhk.edu.hk
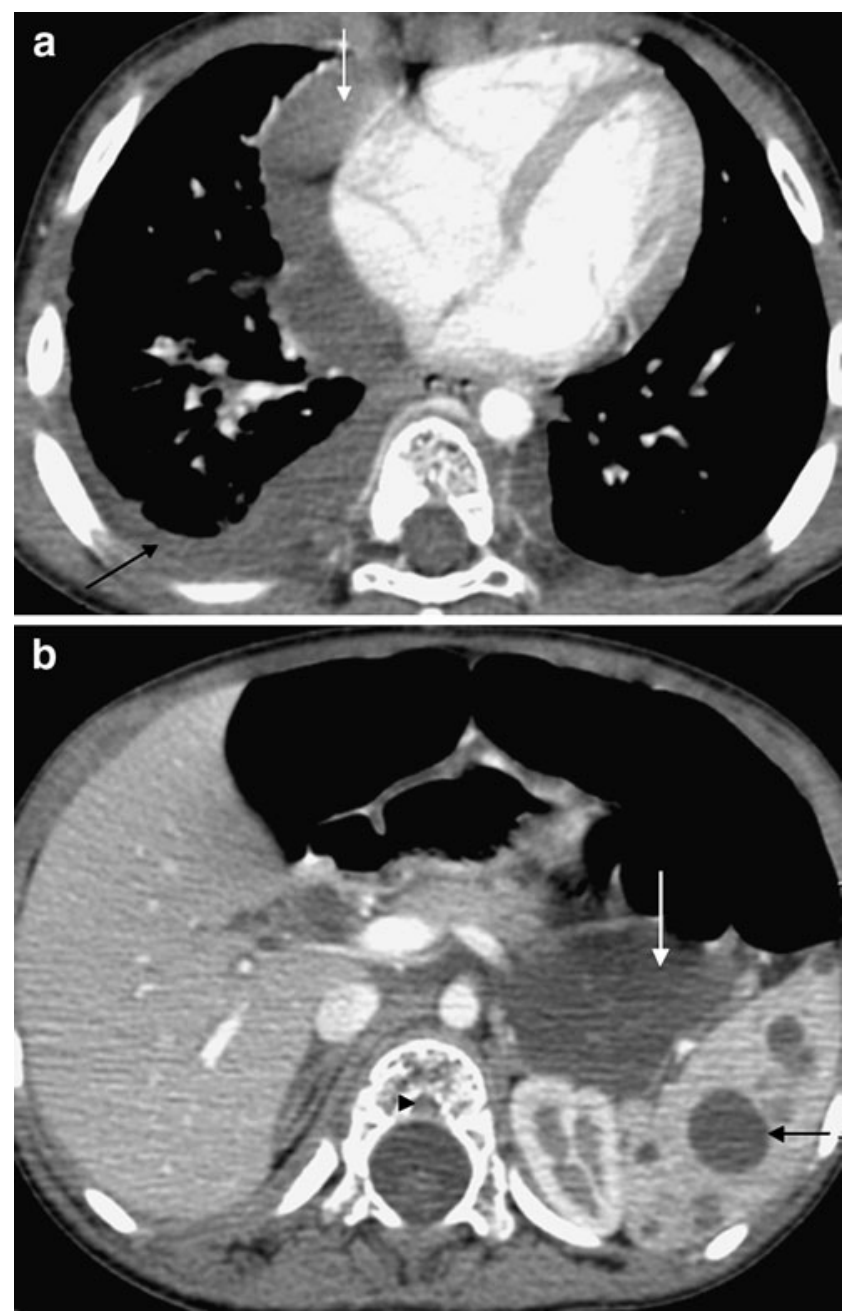

Fig. 1 Contrast-enhanced CT. a Axial thoracic image. b axial abdominal image

\section{References}

1. Brown LR, Reiman HM, Rosenow EC et al (1986) Intrathoracic lymphangioma. Mayo Clin Proc 61:882-892

2. Wunderbaldinger P, Paya K, Partik B et al (2000) CT and MR imaging of generalized cystic lymphangiomatosis in pediatric patients. AJR 174:827-832 